\title{
Quantum Weiss-Weinstein bounds for quantum metrology
}

\author{
Xiao-Ming $\mathrm{Lu}^{1, *}$ and Mankei Tsang ${ }^{1,2, \dagger}$ \\ ${ }^{1}$ Department of Electrical and Computer Engineering, \\ National University of Singapore, 4 Engineering Drive 3, Singapore 117583 \\ ${ }^{2}$ Department of Physics, National University of Singapore, 2 Science Drive 3, Singapore 117551
}

\begin{abstract}
Sensing and imaging are among the most important applications of quantum information science. To investigate their fundamental limits and the possibility of quantum enhancements, researchers have for decades relied on the quantum Cramér-Rao lower error bounds pioneered by Helstrom. Recent work, however, has called into question the tightness of those bounds for highly nonclassical states in the non-asymptotic regime, and better methods are now needed to assess the attainable quantum limits in reality. Here we propose a new class of quantum bounds called quantum WeissWeinstein bounds, which include Cramér-Rao-type inequalities as special cases but can also be significantly tighter to the attainable error. We demonstrate the superiority of our bounds through the derivation of a Heisenberg limit and phase-estimation examples.
\end{abstract}

\section{INTRODUCTION}

Quantum noise is becoming a major limiting factor in sensing and imaging technology, with photon shot noise in particular imposing limits to modern gravitationalwave detectors [1] as well as optical microscopes [2]. Quantum metrology [3, 4], through the use of nonclassical states or innovative measurement schemes, promises to beat such conventional quantum limits and offer significant accuracy enhancements. This promise has led to renewed interest in the quantum estimation theory pioneered by Helstrom [5], and especially the quantum Cramér-Rao bounds (QCRBs) [5-7]. The bounds were originally developed to investigate thermal and laser sources, but they are now being applied to increasingly exotic quantum states for the purpose of quantum enhancements. The asymptotic attainability of Helstrom's QCRB for one parameter [8, 9] and Holevo's version for multiple parameters [10] suggests that the QCRBs can be tight; many proposals of quantum enhancements are based just on QCRBs [11-14]. Unfortunately, these works ignore the number of repeated trials needed to reach the asymptotic regime, and the requirement of many repetitions can negate the perceived advantage of their protocols, as realized by subsequent studies [15-23].

These mishaps suggest that Helstrom's paradigm of quantum estimation theory can no longer fulfill the modern demands of quantum metrology and better approaches are needed to assess quantum sensors in highly nonclassical states. We can take inspiration from classical estimation theory, where it is common knowledge that Cramér-Rao-type inequalities can grossly underestimate the attainable estimation error [24]. Two new families of bounds have emerged there as the best candidates to supersede the Cramér-Rao family [24]: the Ziv-Zakai family [25] and the Weiss-Weinstein family [26, 27]. Although they are derived from distinct principles, both have been

\footnotetext{
* luxiaoming@gmail.com

$\dagger$ mankei@nus.edu.sg
}

found to remain remarkably tight to the attainable estimation errors in both non-asymptotic and asymptotic regimes, with diverse applications in engineering [24] as well as astronomy [28]. For the quantum problem, quantum Ziv-Zakai bounds (QZZBs) have recently been proposed and shown to be superior to QCRBs in many cases [15-17]. Although the QZZBs are trivial to prove and straightforward to evaluate, there is no general guarantee about their superiority over the QCRBs, so they have to be compared on a case-by-case basis. To overcome this problem, here we propose quantum versions of the Weiss-Weinstein bounds, which have the advantage of including Cramér-Rao-type inequalities as special cases. Through the derivation of a Heisenberg limit and examples of phase estimation, we further demonstrate that our new bounds can not only beat QCRBs but also QZZBs for tightness.

\section{RESULTS}

\section{A. Quantum covariance inequality}

Our quantum Weiss-Weinstein bounds (QWWBs) are based on a quantum generalization of the covariance inequality proposed by Weinstein and Weiss $[24,27]$. It is a lower bound on the global estimation error matrix defined as

$$
\Sigma:=\int d x d y \epsilon(x, y) \epsilon(x, y)^{\top} p(x, y),
$$

where $x \in \mathbb{R}^{J}$ is a column vector of $J$ unknown parameters, $y$ is the observation, $p(x, y)$ is their joint probability distribution, $\epsilon(x, y):=\tilde{x}(y)-x$ is the error vector with respect to an estimator $\tilde{x}(y)$, and $\top$ denotes the matrix transpose. For the quantum problem [7],

$$
p(x, y)=\operatorname{Tr}\left[E_{y} \rho(x)\right],
$$

where $\rho(x)=\rho_{x} p(x)$ is the hybrid density operator [29], $\rho_{x}$ is the conditional density operator that models the quantum system as a function of $x, p(x)$ is the prior 
distribution, $E_{y}$ is the positive operator-valued measure (POVM) that models the quantum measurement $[5,6]$, and $\operatorname{Tr}$ denotes the operator trace. Our quantum covariance inequality reads

$$
\Sigma \geq C G^{-1} C^{\top},
$$

where $G$ is a $K \times K$ real and strictly positive matrix defined as

$$
G_{k k^{\prime}}:=\int d x \operatorname{Re} \operatorname{Tr}\left[L_{k}(x)^{\dagger} L_{k^{\prime}}(x) \rho(x)\right]
$$

in terms of a set of operators $\left\{L_{k}(x) ; k=1,2, \ldots, K\right\}$ and $C$ is a $J \times K$ real matrix defined as

$$
C_{j k}:=\int d x d y \epsilon_{j}(x, y) \operatorname{Re} \operatorname{Tr}\left[E_{y} L_{k}(x) \rho(x)\right] .
$$

Equation (3) means that $\Sigma-C G^{-1} C^{\top}$ is positivesemidefinite. The proof of Eq. (3) is given in the Methods. To derive measurement-independent quantum bounds, we will choose a set of $L_{k}(x)$ 's to make $C$ independent of the POVM and the estimator.

\section{B. Quantum Weiss-Weinstein bounds}

Our QWWBs posit that each $L_{k}(x)$ satisfies

$$
\begin{aligned}
D_{k}(x) & =\frac{1}{2}\left[L_{k}(x) \rho(x)+\rho(x) L_{k}(x)^{\dagger}\right], \\
D_{k}(x) & :=\frac{V_{k}\left(x+h_{k}\right)-V_{k}(x)}{\left|h_{k}\right|} \\
V_{k}(x) & :=\mathcal{N}_{k} \rho(x)^{s_{k}} \circ \rho\left(x-h_{k}\right)^{1-s_{k}}
\end{aligned}
$$

where $h_{k}$ is a real vector with length $\left|h_{k}\right|$ and the same dimension as that of $x, 0<s_{k}<1, O_{1} \circ O_{2}:=$ $\left(O_{1} O_{2}+O_{2} O_{1}\right) / 2$ denotes the Jordan product, and $\mathcal{N}_{k}$ is a normalization factor such that $\int d x \operatorname{Tr} V_{k}(x)=1$. This choice of $L_{k}(x)$ gives

$$
C_{j k}=\frac{h_{k j}}{\left|h_{k}\right|},
$$

where $h_{k j}$ is the $j$ th component of $h_{k}$. To see this, notice that, with $s_{k}$ being set in the range $(0,1), V_{k}(x)$ vanishes where $p(x)$ vanishes, leading to $\int d x V_{k}(x+h)=$ $\int d x V_{k}(x)$ and $\int d x D_{k}(x)=0$, as the domain of integration is $\mathbb{R}^{J}$ and $p(x)$ must vanish at infinity. It then follows from Eqs. (5) and (6) and the completeness property of $E_{y}$ that $C_{j k}=-\int d x x_{j} \operatorname{Tr}\left[V_{k}(x+h)-V_{k}(x)\right] /\left|h_{k}\right| . \mathrm{A}$ change of variables gives $\int d x x_{j} V_{k}\left(x+h_{k}\right)=\int d x\left(x_{j}-\right.$ $\left.h_{k j}\right) V_{k}(x)$, which leads to Eq. (9).

The QWWBs given by Eqs. (3)-(9) are applicable to any quantum measurement, any biased or unbiased estimator, and do not require $\rho_{x}$ or $p(x)$ to be differentiable. They are a family of bounds that hold for any $K$, any $h_{k}$, and any $0<s_{k}<1$, such that tighter versions can be obtained by choosing these parameters judiciously. The $\left|h_{k}\right| \rightarrow 0$ limit leads to the Bayesian QCRBs $[7,29]$ (see Appendix B), while finite $h_{k}$ and $s_{k} \rightarrow 1$ lead to Bayesian multiparameter versions of the quantum bounds proposed by Tsuda and Matsumoto [30]. The classical Weiss-Weinstein bound is usually computed with $s_{k}=1 / 2$ since it often maximizes the bound [24, 26, 27]; our examples later show that $s_{k}=1 / 2$ can also lead to tight quantum bounds.

The $L_{k}(x)$ operators may not be uniquely determined by Eq. (6) for a given $\rho(x)$ and $D_{k}(x)$. We prove in Appendix $\mathrm{C}$ that the Hermitian $L_{k}(x)$ 's give the tightest QWWB, though non-Hermitian choices may be easier to obtain in some cases. When $\rho(x)$ and $D_{k}(x)$ are of low rank, the following expression is useful to obtain the Hermitian $L_{k}(x)$ 's:

$$
L_{k}(x)=\sum_{\alpha, \beta \mid \lambda_{\alpha}+\lambda_{\beta} \neq 0} \frac{2\left\langle\alpha\left|D_{k}(x)\right| \beta\right\rangle}{\lambda_{\alpha}+\lambda_{\beta}}|\alpha\rangle\langle\beta|,
$$

where each $|\alpha\rangle$ is an eigenstate of $\rho(x)$ with eigenvalue $\lambda_{\alpha}$. Taking $D_{k}(x)$ as the partial derivative with respect to $x_{k}$, Eq. (10) is a well-known expression for the symmetric logarithmic derivative operator [5, 31]. For nonHermitian $L_{k}(x)$ 's, the QWWB can be tightened by noting that $L_{k}(x)+i \alpha_{k}$, with $\alpha_{k}$ being an arbitrary real number, is also a solution of Eq. (6). Maximizing the positive matrix $G$ over $\alpha_{k}$ leads to $\alpha_{k}=-\operatorname{Im}\left\langle L_{k}(x)\right\rangle$, where $\langle\bullet\rangle:=\int d x \operatorname{Tr}[\bullet \rho(x)]$. We can therefore always replace $G$ by $G-\Delta$ to tighten the QWWBs, where $\Delta_{k k^{\prime}}=\operatorname{Im}\left\langle L_{k}(x)\right\rangle \operatorname{Im}\left\langle L_{k^{\prime}}(x)\right\rangle$ is a positive-semidefinite matrix.

The QWWBs degenerates into the classical WeissWeinstein bounds $[24,26,27]$ for a commuting family of $\rho_{x}$. In such a situation, we can identify a basis $\{|y\rangle\}$ in which all $\rho_{x}$ are diagonal matrices, meaning that $\rho(x)$ can be equivalently expressed as a joint probability $p(x, y):=\langle y|\rho(x)| y\rangle$. Consequently, $L_{k}(x)$ is also diagonal with the basis $\{|y\rangle\}$, and can be expressed as a function

$L_{k}(x, y)=\frac{\mathcal{N}_{k}}{\left|h_{k}\right|}\left\{\left[\frac{p\left(x+h_{k}, y\right)}{p(x, y)}\right]^{s_{k}}-\left[\frac{p\left(x-h_{k}, y\right)}{p(x, y)}\right]^{1-s_{k}}\right\}$,

where $\mathcal{N}_{k}=\mathbb{E}\left[p\left(x+h_{k}, y\right)^{s_{k}} / p(x, y)^{s_{k}}\right]^{-1}$, and $\mathbb{E}[\bullet]$ denotes the expectation value with respect to the joint probability $p(x, y)$. The Classical Weiss-Weinstein bound is still of the form Eq. (3) with $C$ being given by Eq. (9), whereas $G$ is expressed in a classical manner as $G_{k k^{\prime}}=$ $\mathbb{E}\left[L_{k}(x, y) L_{k^{\prime}}(x, y)\right]$.

\section{Single-parameter estimation}

For single-parameter estimation, the error matrix reduces to the mean-square error $\Sigma=\int d x d y[\tilde{x}(y)-$ $x]^{2} p(x, y)$. The QWWBs become

$$
\Sigma \geq \Sigma_{\mathrm{W}}(s, h):=\frac{1}{\left\langle L(x)^{\dagger} L(x)\right\rangle-[\operatorname{Im}\langle L(x)\rangle]^{2}} .
$$


The following choice of $L(x)$ serves our purpose:

$$
L(x)=\frac{\mathcal{N}(s, h)}{|h|}[\Lambda(s, h)-\Lambda(1-s,-h)],
$$

where $\Lambda(s, h):=\rho(x+h)^{s} \rho(x)^{-s}$ and $\mathcal{N}(s, h)=$ $\langle\Lambda(s, h)\rangle^{-1}$. Here we use the convention that a power of a positive-semidefinite operator is taken only on its support [32]. $\rho(x)^{-1}$ is then the generalized inverse defined on the support and $\rho(x)^{0}$ is the projector onto the support. Consequently, $L(x)$ vanishes where $p(x)$ vanishes for $0<s<1$. Equation (12) becomes

$$
\Sigma_{\mathrm{W}}(s, h)=\frac{h^{2} g(s, h)^{2}}{g(2 s, h)+g(2-2 s,-h)-2 \tilde{g}(s, 2 h)},
$$

where $g(s, h) \quad:=\langle\Lambda(s, h)\rangle$ and $\tilde{g}(s, 2 h) \quad:=$ $\operatorname{Re}\left\langle\Lambda(s, h)^{\dagger} \Lambda(1-s,-h)\right\rangle$. When the conditional density operators $\rho_{x}$ are of full rank, it can be shown that $\tilde{g}(s, h)=g(s, h)$. Equation (14) is then of the same form as the classical Weiss-Weinstein bound [26], but with a different function $g(s, h)$. Although the characteristics of $g(s, h)$ determines the QWWB in an intricate manner, some intuitive observations can be given as follows. The situation of particular interest is that $\Sigma_{\mathrm{W}}(s, h)$ takes its maximum at a finite large value of $h$ rather than at $h \rightarrow 0$, meaning that the QCRB underestimates the error. For the case of $\tilde{g}(s, h)=g(s, h)$, the denominator of Eq. (14) is bounded above by 2 due to $g(s, h) \in[0,1]$. Then, considering the factor $h^{2}$ in the numerator, Eq. (14) may take its maximum at a finite large value of $h$, when $g(s, h)$ is not always far less than one as $h$ becomes large. The estimation models with such a characteristic of $g(s, h)$ may be poorly assessed by only the QCRB, thereby are in need of the QWWB or the QZZB.

We now focus on phase estimation, a paradigmatic problem in quantum metrology. Assume $\rho_{x}=$ $\exp (-i x H) \rho \exp (i x H)$, where $\rho$ is the initial state and $H$ is an Hermitian operator. In this case, $g(s, h)$ and $\tilde{g}(s, h)$ can be neatly separated as $g(s, h)=g_{\mathrm{c}}(s, h) g_{\mathrm{q}}(s, h)$ and $\tilde{g}(s, h)=g_{\mathrm{c}}(s, h) \tilde{g}_{\mathrm{q}}(s, h)$, where

$$
g_{\mathrm{c}}(s, h)=\int_{\{x ; p(x)>0\}} d x p(x+h)^{s} p(x)^{1-s}
$$

is a classical component that depends only on the prior, and

$$
\begin{aligned}
g_{\mathrm{q}}(s, h) & =\operatorname{Tr}\left(\rho_{h}^{s} \rho^{1-s}\right), \\
\tilde{g}_{\mathrm{q}}(s, 2 h) & =\operatorname{Re} \operatorname{Tr}\left(\rho_{h}^{s} \rho_{-h}^{1-s} \rho^{0}\right)
\end{aligned}
$$

are quantum components. If the initial state is pure, $\rho=|\psi\rangle\langle\psi|$, and since $\rho^{s}=\rho$ for a pure state, we obtain $g_{\mathrm{q}}(s, h)=|z(h)|^{2}$ and $\tilde{g}_{\mathrm{q}}(s, 2 h)=\operatorname{Re} z(h)^{2} z(2 h)^{*}$, where $z(h):=\langle\psi|\exp (-i h H)| \psi\rangle$. Interestingly, the quantity $g_{\mathrm{q}}(s, h)$ also plays an important role in the quantum Chernoff bound for binary hypothesis testing [33-35], although no meaningful relationship between the WeissWeinstein bound and the Chernoff bound, apart from the coincidental mathematical similarity, has been discovered to our knowledge.

\section{Heisenberg limit}

The QWWBs can be used to derive a Heisenberg limit as follows. Let $|\psi\rangle=\sum_{j} c_{j}|j\rangle$ be a purification of the initial quantum state, where each $|j\rangle$ is an eigenvector of $H$ with eigenvalue $E_{j}$. Then $\tilde{g}_{\mathrm{q}}(s, 2 h)=$ $\sum_{j k l}\left|c_{j} c_{k} c_{l}\right|^{2} \cos \left[h\left(E_{j}+E_{k}-2 E_{l}\right)\right]$. The cosine function can be bounded as $\cos \theta \geq 1-\lambda|\theta|$, where $\lambda \approx 0.7246$ is the implicit solution of $\lambda=\sin \phi=(1-\cos \phi) / \phi[15]$. Thus, $\tilde{g}_{\mathrm{q}}(s, 2 h) \geq 1-\lambda|h| \sum_{j k l}\left|c_{j} c_{k} c_{l}\right|^{2}\left|E_{j}+E_{k}-2 E_{l}\right|$. Let $E_{0}$ be the minimum eigenvalue of $H$ and $\Delta E_{j}:=$ $E_{j}-E_{0}$. By noting that $\left|E_{j}+E_{k}-2 E_{l}\right|=\mid \Delta E_{j}+$ $\Delta E_{k}-2 \Delta E_{l} \mid \leq \Delta E_{j}+\Delta E_{k}+2 \Delta E_{l}$, it follows that $\tilde{g}_{\mathrm{q}}(s, 2 h) \geq 1-4 \lambda|h| H_{+}$with $H_{+}:=\operatorname{Tr}(H \rho)-E_{0}$. Consequently, $\tilde{g}_{\mathrm{q}}(s, 2 h)$ is nonnegative when $|h| \leq 1 /\left(4 \lambda H_{+}\right)$; this implies that the QWWB is further bounded as

$$
\begin{aligned}
\Sigma \geq \Sigma_{\mathrm{W}}^{\prime}(h) & :=\kappa(h) h^{2}|z(h)|^{2} \text { with } \\
\kappa(h) & :=\sup _{0<s<1} \frac{g_{\mathrm{c}}(s, h)^{2}}{g_{\mathrm{c}}(2 s, h)+g_{\mathrm{c}}(2-2 s,-h)}
\end{aligned}
$$

for $|h| \leq h_{\star}:=1 /\left(4 \lambda H_{+}\right)$. The quantity $|z(h)|^{2}$ is the quantum fidelity between $|\psi\rangle$ and $\exp (-i h H)|\psi\rangle$, which is bounded as $|z(h)|^{2} \geq 1-\left|2 h \lambda H_{+}\right|[15]$. Taking $h=h_{\star}$, one obtains

$$
\Sigma \geq \Sigma_{\mathrm{W}}\left(h_{\star}\right)^{\prime} \geq \frac{\kappa\left(h_{\star}\right)}{32 \lambda^{2} H_{+}^{2}} .
$$

We have not yet made any assumption about the prior, which is incorporated in $\kappa\left(h_{\star}\right)$. Since $g_{\mathrm{c}}(1 / 2, \pm h) \leq 1$, it follows that $\kappa(h) \geq g_{\mathrm{c}}(1 / 2, h)^{2} / 2$. The quantity $g_{\mathrm{c}}(1 / 2, h)$, also known as the Bhattacharyya coefficient, measures the overlap between the prior probability distribution $p(x)$ and its displaced version $p(x+h)$. For a large enough $H_{+}$(corresponding to a small enough $h_{\star}$ ) such that $g_{\mathrm{c}}\left(1 / 2, h_{\star}\right) \approx 1$, Eq. (19) gives a Heisenberg limit as $1 /\left(64 \lambda^{2} H_{+}^{2}\right)$, which is higher than the limit $1 /\left(80 \lambda^{2} H_{+}^{2}\right)$ derived from a QZZB in Ref. [15]. Both this work and Ref. [15] use a linear lower bound on the fidelity; an even tighter Heisenberg limit can be obtained via the stronger fidelity bound in Ref. [20]. For a generator $H$ with integer eigenvalues, a stronger Heisenberg limit was derived through some information-theoretic inequalities [21, 22].

\section{E. Phase-estimation examples}

We now demonstrate the tightness of QWWBs relative to other existing quantum bounds through two examples. The first example is the estimation of a random phase with Gaussian prior via a qubit. Assume that the initial qubit state is $|\psi\rangle=(|0\rangle+|1\rangle) / \sqrt{2}$, the generator is $H=$ $E|1\rangle\langle 1|$ with $E>0$, and the standard deviation of the prior is $\sigma$. For this simple model, the minimum meansquare error (MMSE) can be analytically calculated [36, 37], and we can use it as a benchmark for the quantum 


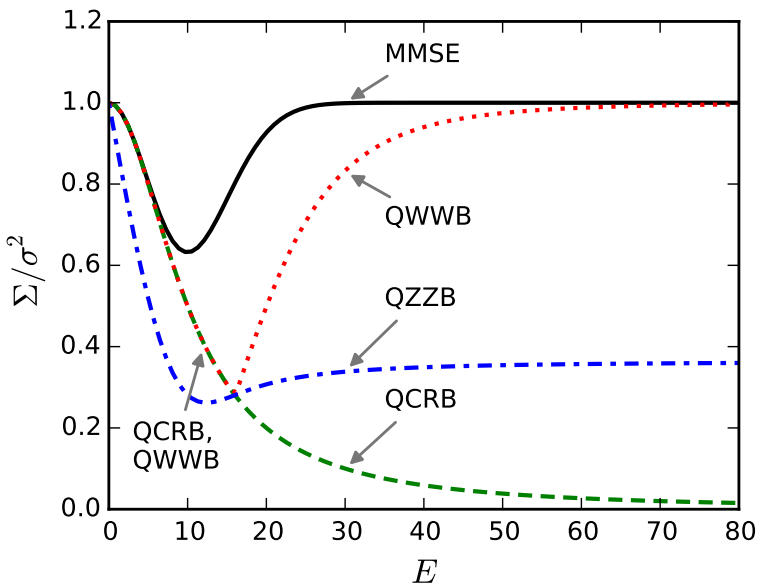

FIG. 1. Comparison of the MMSE (black solid), QWWB (red dotted), QZZB (blue dash-dotted), and the Bayesian QCRB (green dashed) for the estimation of a random phase via a qubit. The prior distribution is Gaussian with $\sigma=0.1$ standard deviation. The QWWB is numerically optimized over $h \in[0,10 \sigma]$ while $s$ is set to $1 / 2$. The MMSE and the error bounds are normalized with respect to the prior value $\sigma^{2}$.

bounds. Setting $s=1 / 2$, the QWWB is given by

$$
\Sigma \geq \sup _{h} \frac{h^{2} \exp \left[-h^{2} /\left(4 \sigma^{2}\right)\right] \cos (h E / 2)^{2}}{2-2 \exp \left[-h^{2} /\left(2 \sigma^{2}\right) \cos (h E)\right]},
$$

see Appendix D for details. Since $\exp (-i x H)|\psi\rangle$ has a period of $2 \pi / E, x$ and $x+2 \pi / E$ are fundamentally indistinguishable from any quantum measurement. This ambiguity means that even the optimal measurement can produce an estimate in the wrong period, leading to substantial errors. The MMSE stays close to the prior value $\sigma^{2}$ as a result, as shown in Fig. 1. The QCRB, on the other hand, is incapable of accounting for the phase ambiguity because of its differential nature and severely underestimates the attainable error for large $E$. The QZZB is not much better, and the QWWB, being close to the QCRB where it is reasonably tight and also following the MMSE for larger $E$, is the clear winner in this benchmark example.

For the second example, we consider phase estimation using $\nu$ independent and identically distributed bosonic probes. For each probe, we assume $H=\sum_{j=0}^{\infty} j|j\rangle\langle j|$ and $|\psi\rangle=\sqrt{1-\epsilon}|0\rangle+\sqrt{\epsilon / M} \sum_{j=1}^{M}|j\rangle$, with $M \geq 1$ being an integer and $0<\epsilon<1$ [12]. In this case the MMSE is not known, and we have to rely on quantum bounds to investigate the fundamental limit. Figure 2 compares the three quantum bounds for $\epsilon=0.1$ and $M=10$. Though the asymptotic attainability of the QCRB $[8,9]$ means that it should be tight for large enough $\nu$, the QCRB by itself is incapable of determining the $\nu$ needed for tightness. It is remarkable that the QWWB and the QZZB, though derived from different principles, follow similar behaviors here. Both are substantially higher than the

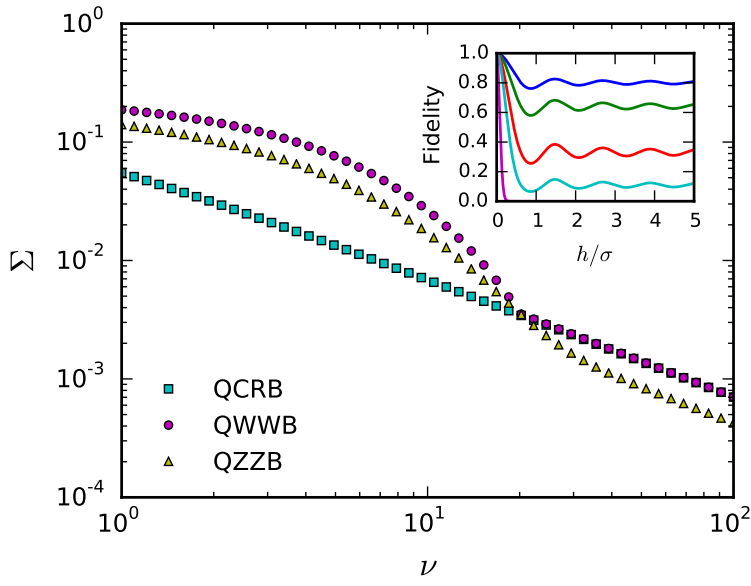

FIG. 2. Error bounds versus the number $\nu$ of identically distributed quantum bosonic probes. The prior distribution is Gaussian with $\sigma=0.5$ standard deviation. The QWWB is numerically optimized over $h \in[0,10 \sigma]$ with $s=1 / 2$, and the QZZB is computed according to Ref. [15]. The inset plots the fidelity $|\langle\psi|\exp (-i h H)| \psi\rangle|^{2 \nu}$ for $\nu=1,2,5,10,100$ (from above to below).

QCRB for small $\nu$ and demonstrate a threshold behavior as $\nu$ is increased, revealing the regime where the prior information dominates and the QCRB is overly optimistic. Once again, the QWWB is higher than the other bounds for all values of $\nu$.

\section{F. Multiple test points}

Similar to the classical Weiss-Weinstein family of error bounds [26, 27], the quantum bounds can be tightened by involving multiple test points. As an example, consider the QWWB for a single-parameter estimation with two test points, for which $G$ is a $2 \times 2$ matrix whose inverse can be explicitly expressed as

$$
G^{-1}=\frac{1}{\operatorname{det} G}\left(\begin{array}{cc}
G_{22} & -G_{12} \\
-G_{21} & G_{11}
\end{array}\right) .
$$

Since $C=(1,1)$, the lower bound from Eq. (3) becomes

$$
\begin{aligned}
& C G^{-1} C^{\top}=\frac{G_{11}+G_{22}-G_{12}-G_{21}}{\operatorname{det} G} \\
& \geq \max \left\{\frac{1}{G_{11}}, \frac{1}{G_{22}}\right\},
\end{aligned}
$$

where the inequality is due to the fact that $G$ is symmetric and positive, meaning that the two-test-point lower bound is tighter than that given by either of the two test points. Following the same strategy that derives the combined Bayesian bound in classical parameter estimation [24], we can set the first test point $h_{1}$ to an infinitesimal value and the second test point $h_{2}$ to a finite value, leading to a combined quantum error bound tighter than the QCRB. 


\section{DISCUSSION}

Our QWWBs set a higher standard in quantum metrology. Not only do they include QCRBs as special cases and inherit their asymptotic tightness at least for one parameter $[8,9]$, they can also beat the recently invented QZZBs [15] and serve as the more natural successors of the Cramér-Rao family in the post-Helstrom era of quantum metrology. Our results demonstrate that differential geometry of quantum states alone [31, 38] cannot guarantee their usefulness; more general distance measures, such as the quantum Chernoff distance used in our QWWBs and the trace distance in the QZZBs, should be consulted to establish tighter quantum limits to parameter estimation, especially for nonclassical states or nontrivial parameter dependence. Future proposals of quantum metrological schemes should no longer rely only on QCRBs to support their cases without also investigating their tightness. We envision our QWWBs to be the new standard against which these proposals should be assessed.

\section{ACKNOWLEDGMENTS}

We acknowledge helpful discussions with Ranjith Nair, Shan Zheng Ang, and Shilin Ng. This work is supported by the Singapore National Research Foundation under NRF Grant No. NRF-NRFF2011-07 and Singapore Ministry of Education Academic Research Fund Tier 1 Project R-263-000-C06-112.

\section{CONTRIBUTIONS}

X.-M. L. invented the quantum Weiss-Weinstein bounds presented here and performed all the proofs and calculations. M. T. conceived the problem. Both authors discussed extensively during the course of this work and contributed to the writing of the manuscript.

\section{Appendix A: Proof of the quantum covariance inequality}

Here we prove Eq. (3). Let $u$ and $v$ be arbitrary real column vectors of dimension $J$ and $K$ respectively. It follows from the definitions that

$$
\begin{aligned}
& u^{\top} \Sigma u=\int d x d y \epsilon_{u}(x, y)^{2} \operatorname{Tr}\left(A^{\dagger} A\right), \\
& v^{\top} G v=\int d x d y \operatorname{Tr}\left(B^{\dagger} B\right),
\end{aligned}
$$

where $\epsilon_{u}(x, y):=\sum_{j} u_{j} \epsilon_{j}(x, y), A:=\sqrt{E_{y}} \sqrt{\rho(x)}$, and $B:=\sqrt{E_{y}}\left[\sum_{k} v_{k} L_{k}(x)\right] \sqrt{\rho(x)}$. In Eq. (A2), we have used $\int d y E_{y}=I$ with $I$ being the identity operator. As a result of the Cauchy-Schwarz inequality,

$$
\sqrt{u^{\top} \Sigma u v^{\top} G v} \geq \int d x d y\left|\epsilon_{u}(x, y)\right| \sqrt{\operatorname{Tr}\left(A^{\dagger} A\right) \operatorname{Tr}\left(B^{\dagger} B\right)} .
$$

From the inequality $\operatorname{Tr}\left(A^{\dagger} A\right) \operatorname{Tr}\left(B^{\dagger} B\right) \geq\left|\operatorname{Tr}\left(A^{\dagger} B\right)\right|^{2}$ followed by $\left|\operatorname{Tr}\left(A^{\dagger} B\right)\right| \geq\left|\operatorname{Re} \operatorname{Tr}\left(A^{\dagger} B\right)\right|$, we get

$$
\begin{gathered}
\left(u^{\top} \Sigma u\right)\left(v^{\top} G v\right) \geq\left[\int d x d y\left|\epsilon_{u}(x, y) \operatorname{Re} \operatorname{Tr}\left(A^{\dagger} B\right)\right|\right]^{2} \\
\geq\left|\int d x d y \epsilon_{u}(x, y) \operatorname{Re} \operatorname{Tr}\left(A^{\dagger} B\right)\right|^{2}=\left(u^{\top} C v\right)^{2} .
\end{gathered}
$$

Taking $v=G^{-1} C^{\top} u$ implies $\left(u^{\top} \Sigma u\right)\left(u^{\top} C G^{-1} C^{\top} u\right) \geq$ $\left(u^{\top} C G^{-1} C^{\top} u\right)^{2}$. Since $G$ is strictly positive, $u^{\top} C G^{-1} C^{\top} u$ is positive, leading to $u^{\top} \Sigma u \geq$ $u^{\top} C G^{-1} C^{\top} u$. As this inequality holds for any real vector $u$, Eq. (3) results.

\section{Appendix B: Relation between QWWB and QCRB}

We here show that the QWWBs include the QCRB as a special case. Let $h_{k}$ be along the direction of $x_{k}$ in the parameter vector space. Suppose that $\rho(x)$ is differentiable. When $\left|h_{k}\right| \rightarrow 0$, one has

$$
\rho\left(x+h_{k}\right)^{s_{k}} \simeq \rho(x)^{s_{k}}+\left|h_{k}\right| \partial \rho(x)^{s_{k}} / \partial x_{k}
$$

It can be shown from Eqs. (7), (8) and (B1) that $D_{k}(x) \simeq$ $\mathcal{N}_{k} \partial \rho(x) / \partial x_{k}$, where the normalizing factor $\mathcal{N}_{k}^{-1}$ can be given by

$$
\mathcal{N}_{k}^{-1} \simeq 1-\left|h_{k}\right| \int d x \operatorname{Tr}\left[\rho(x)^{s_{k}} \frac{\partial \rho(x)^{1-s_{k}}}{\partial x_{k}}\right] .
$$

Let $\rho(x)=\sum_{\alpha} \lambda_{\alpha}\left|\phi_{\alpha}\right\rangle\left\langle\phi_{\alpha}\right|$ be the eigenvalue decomposition. Since $\rho(x)^{s_{k}}=\sum_{\alpha} \lambda_{\alpha}^{s_{k}}\left|\phi_{\alpha}\right\rangle\left\langle\phi_{\alpha}\right|$, it follows that

$$
\begin{aligned}
& \int d x \operatorname{Tr}\left[\rho(x)^{s_{k}} \frac{\partial \rho(x)^{1-s_{k}}}{\partial x_{k}}\right] \\
= & \sum_{\alpha} \int d x\left[\lambda_{\alpha}^{s_{k}} \frac{\partial \lambda_{\alpha}^{1-s_{k}}}{\partial x_{k}}+\lambda_{\alpha}\left(\left\langle\phi_{\alpha} \mid \frac{\partial \phi_{\alpha}}{\partial x_{k}}\right\rangle+\left\langle\frac{\partial \phi_{\alpha}}{\partial x_{k}} \mid \phi_{\alpha}\right\rangle\right)\right] \\
= & \left(1-s_{k}\right) \sum_{\alpha} \int d x \frac{\partial \lambda_{\alpha}}{\partial x_{k}}+\sum_{\alpha} \int d x \lambda_{\alpha} \frac{\partial}{\partial x_{k}}\left\langle\phi_{\alpha} \mid \phi_{\alpha}\right\rangle \\
= & 0,
\end{aligned}
$$

where we have used $\left.\lambda_{\alpha}\right|_{x_{k}= \pm \infty}=0$ in the last equality. Thus, $D_{k}(x) \simeq \partial \rho(x) / \partial x_{k}$ when $\left|h_{k}\right| \rightarrow 0$. Consequently, the operator $L_{k}(x)$ becomes the symmetric logarithmic derivative operator (not necessarily to be Hermitian, see Ref. [29]) for $\rho(x)$ with respect to $x_{k}$, and the resulting QWWB becomes a corresponding QCRB. 


\section{Appendix C: Hermitian $L_{k}(x)$ tightening the QWWB}

Here, we prove that the Hermitian $L_{k}(x)$ gives the tightest lower bound on the estimation-error covariance matrix among all choices of $L_{k}(x)$ satisfying Eq. (6) for given $\rho(x)$ and $D_{k}(x)$. This can be seen from the following Proposition.

Proposition. Suppose that $L$ is an operator satisfying

$$
\frac{1}{2}\left(L \rho+\rho L^{\dagger}\right)=D
$$

where $\rho$ is a given positive semidefinite operator and $D$ is a given Hermitian operator. Then,

$$
\min \operatorname{Tr}\left(L^{\dagger} L \rho\right)=\operatorname{Tr}\left(\tilde{L}^{\dagger} \tilde{L} \rho\right)
$$

where the minimum is taken over all solutions of Eq. (C1) for $L$, and $\tilde{L}$ denotes a Hermitian solution.

Proof. Let $M=\left(L+L^{\dagger}\right) / 2$ and $N=\left(L-L^{\dagger}\right) /(2 i)$, which are both Hermitian operators. Let $\rho=\sum_{j} \lambda_{j}|j\rangle\langle j|$ be the eigenvalue decomposition. It follows from Eq. (C1) and $L=M+i N$ that

$$
D_{j k}=\frac{1}{2}\left(\lambda_{k}+\lambda_{j}\right) M_{j k}+\frac{i}{2}\left(\lambda_{k}-\lambda_{j}\right) N_{j k}
$$

where the elements of the matrices are represented in the basis $\{|j\rangle\}$. This equality implies that we can always freely choose $N$ and determine $M$ accordingly in terms of $D$ and $N$. When $\lambda_{j}+\lambda_{k} \neq 0$, we have

$$
M_{j k}=\frac{2 D_{j k}+i\left(\lambda_{j}-\lambda_{k}\right) N_{j k}}{\lambda_{j}+\lambda_{k}}
$$

which implies that $L_{j k}=\left(2 D_{j k}+2 i \lambda_{j} N_{j k}\right) /\left(\lambda_{j}+\lambda_{k}\right)$. It then follows that

$$
\begin{aligned}
\operatorname{Tr}\left(L^{\dagger} L \rho\right) & =\sum_{j, k \mid \lambda_{k}>0} \lambda_{k}\left|L_{j k}\right|^{2} \\
& =\sum_{j, k \mid \lambda_{k}>0} \frac{4 \lambda_{k}}{\left(\lambda_{j}+\lambda_{k}\right)^{2}}\left|D_{j k}+i \lambda_{j} N_{j k}\right|^{2} \\
& =\sum_{j, k \mid \lambda_{k}>0} A_{j k}+\sum_{j, k \mid \lambda_{j}>0, \lambda_{k}>0} B_{j k},
\end{aligned}
$$

where

$$
\begin{aligned}
A_{j k} & :=\frac{4 \lambda_{k}}{\left(\lambda_{j}+\lambda_{k}\right)^{2}}\left(\left|D_{j k}\right|^{2}+\lambda_{j}^{2}\left|N_{j k}\right|^{2}\right), \\
B_{j k} & :=\frac{4 i \lambda_{j} \lambda_{k}}{\left(\lambda_{j}+\lambda_{k}\right)^{2}}\left(D_{j k}^{*} N_{j k}-D_{j k} N_{j k}^{*}\right) .
\end{aligned}
$$

Since both $D$ and $N$ are Hermitian, we have $D_{j k}^{*}=D_{k j}$ and $N_{j k}^{*}=N_{k j}$, which implies that the matrix $B$ is antisymmetric as $B_{j k}=-B_{k j}$. Therefore,

$$
\operatorname{Tr}\left(L^{\dagger} L \rho\right)=\sum_{j, k \mid \lambda_{k}>0} A_{j k} \geq \sum_{j k \mid \lambda_{j}>0} \frac{4 \lambda_{k}\left|D_{j k}\right|^{2}}{\left(\lambda_{j}+\lambda_{k}\right)^{2}} .
$$

The equality in the above inequality holds when all $N_{j k}$ vanishes, meaning that $L$ is Hermitian.

Now, let us consider the case where each $L_{k}(x)$ may be non-uniquely determined by $D_{k}(x)$ and $\rho(x)$ through Eq. (6). Denote the Hermitian solution for $L_{k}(x)$ by $\tilde{L}_{k}(x)$ and define the matrix $\tilde{G}$ by $\tilde{G}_{k k^{\prime}}=$ $\operatorname{Re} \operatorname{Tr}\left[\tilde{L}_{k}(x)^{\dagger} \tilde{L}_{k^{\prime}}(x) \rho(x)\right]$. Let $u$ be an arbitrary real vector. In terms of the above Proposition with $D=$ $\sum_{k} u_{k} D_{k}(x)$ and $L=\sum_{k} u_{k} L_{k}(x)$, it can be shown that $u^{\top} G u \geq u^{\top} \tilde{G} u$, thus $G \geq \tilde{G}$. Suppose that $G$ is strictly positive, then $G \geq \tilde{G}$ implies $\tilde{G}^{-1} \geq G^{-1}$. Thus, the Hermitian $L_{k}(x)$ give the tightest lower bound in the Weiss-Weinstein family.

\section{Appendix D: Phase-estimation example}

Here, we calculate the MMSE, the QWWB, the QCRB, and the QZZB for the first example in Sec. IIE. For a unitary sensing $U_{x}=\exp (-i x H)$ and a Gaussian prior distribution

$$
p(x)=\frac{1}{\sqrt{2 \pi} \sigma} \exp \left[-\frac{(x-\mu)^{2}}{2 \sigma^{2}}\right],
$$

the MMSE of estimating $x$ is given by [36]

$$
\Sigma_{\min }=\sigma^{2}-\sigma^{4} \mathcal{F}(\bar{\rho}, H),
$$

where $\mathcal{F}(\bar{\rho}, H)$ is the quantum Fisher information about a parameter $\theta$ in the parametric quantum state $U_{\theta} \bar{\rho} U_{\theta}^{\dagger}$, where $\bar{\rho}:=\int_{-\infty}^{\infty} d x p(x) U_{x} \rho U_{x}^{\dagger}$ with $\rho$ being the initial state. With the eigenvalue decomposition $\bar{\rho}=$ $\sum_{j} \lambda_{j}|j\rangle\langle j|$, one has

$$
\mathcal{F}(\bar{\rho}, H)=\sum_{j, k \mid \lambda_{j}+\lambda_{k}>0} \frac{2\left(\lambda_{j}-\lambda_{k}\right)^{2}\left|H_{j k}\right|^{2}}{\lambda_{j}+\lambda_{k}} .
$$

In our example, the initial state is $|\psi\rangle=(|0\rangle+|1\rangle) / \sqrt{2}$ and the generator of the unitary sensing is $H=E|1\rangle\langle 1|$, where $E$ is a positive number. Then, the average state is given by

$$
\bar{\rho}=\frac{1}{2}(|0\rangle\langle 0|+| 1\rangle\langle 1|)+\frac{\gamma}{2}(|0\rangle\langle 1|+| 1\rangle\langle 0|)
$$

with $\gamma:=\exp \left(-E^{2} \sigma^{2} / 2\right)$. The eigenvalues and eigenvectors of $\bar{\rho}$ are $(1 \pm \gamma) / 2$ and $(|0\rangle \pm|1\rangle) / \sqrt{2}$ respectively. It then follows from Eq. (D3) that $\mathcal{F}(\bar{\rho}, H)=\gamma^{2} E^{2}$, which implies

$$
\Sigma_{\min }=\sigma^{2}-\sigma^{4} E^{2} \exp \left(-E^{2} \sigma^{2}\right)
$$

To obtain the QWWB, one only needs $g_{\mathrm{c}}(s, h)=$ $\exp \left[-h^{2} s(1-s) /\left(2 \sigma^{2}\right)\right]$ and $z(h)=\left(1+e^{-i E h}\right) / 2$, with which the QWWB is give by 


$$
\Sigma_{\mathrm{W}}(s, h)=\frac{h^{2} g_{\mathrm{c}}(s, h)^{2}|z(h)|^{4}}{\left[g_{\mathrm{c}}(2 s, h)+g_{\mathrm{c}}(2-2 s,-h)\right]|z(h)|^{2}-2 g_{\mathrm{c}}(s, 2 h) \operatorname{Re} z(h)^{2} z(2 h)^{*}} .
$$

Taking $s=1 / 2$ for simplicity, we obtain the QWWB optimized over $h$ as follows:

$$
\Sigma_{\mathrm{W}}=\sup _{h} \frac{h^{2} \exp \left(-\frac{h^{2}}{4 \sigma^{2}}\right) \cos \left(\frac{h E}{2}\right)^{2}}{2-2 \exp \left(-\frac{h^{2}}{2 \sigma^{2}}\right) \cos (h E)}
$$

After some algebras, the QCRB is given by

$$
\Sigma_{\mathrm{C}}=\frac{1}{1 / \sigma^{2}+E^{2}}
$$

and the QZZB is given by

$$
\Sigma_{\mathrm{Z}}=\frac{1}{2} \int_{0}^{+\infty} d h h \operatorname{erfc}\left(\frac{h}{2 \sqrt{2} \sigma}\right)\left[1-\sqrt{1-|z(h)|^{2}}\right]
$$

where $\operatorname{erfc}(x)=(2 / \sqrt{\pi}) \int_{x}^{+\infty} d t e^{-t^{2}}$ is the complementary error function.
[1] B. P. Abbott et al. (LIGO Scientific Collaboration and Virgo Collaboration), Phys. Rev. Lett. 116, 131103 (2016).

[2] J. B. Pawley, ed., Handbook of Biological Confocal Microscopy (Springer, New York, 2006).

[3] V. Giovannetti, S. Lloyd, and L. Maccone, Science 306, 1330 (2004).

[4] V. Giovannetti, S. Lloyd, and L. Maccone, Nat. Photon. 5, 222 (2011).

[5] C. W. Helstrom, Quantum Detection and Estimation Theory (Academic Press, New York, 1976).

[6] A. S. Holevo, Probabilistic and Statistical Aspects of Quantum Theory (North-Holland, Amsterdam, 1982).

[7] H. Yuen and M. Lax, IEEE Trans. Inform. Theory 19, 740 (1973).

[8] M. Hayashi, ed., Asymptotic Theory of Quantum Statistical Inference: Selected Papers (World Scientific, Singapore, 2005).

[9] A. Fujiwara, J. Phys. A: Math. Gen. 39, 12489 (2006).

[10] R. D. Gill and M. I. Guţă, "On asymptotic quantum statistical inference," in From Probability to Statistics and Back: High-Dimensional Models and Processes A Festschrift in Honor of Jon A. Wellner, Collections, Vol. 9, edited by M. Banerjee, F. Bunea, J. Huang, V. Koltchinskii, and M. H. Maathuis (Institute of Mathematical Statistics, Beachwood, Ohio, USA, 2013) pp. $105-127$.

[11] P. M. Anisimov, G. M. Raterman, A. Chiruvelli, W. N. Plick, S. D. Huver, H. Lee, and J. P. Dowling, Phys. Rev. Lett. 104, 103602 (2010).

[12] A. Rivas and A. Luis, New J. Phys. 14, 093052 (2012).

[13] Y. R. Zhang, G. R. Jin, J. P. Cao, W. M. Liu, and H. Fan, J. Phys. A: Math. Theor. 46, 035302 (2013).

[14] P. C. Humphreys, M. Barbieri, A. Datta, and I. A. Walmsley, Phys. Rev. Lett. 111, 070403 (2013).

[15] M. Tsang, Phys. Rev. Lett. 108, 230401 (2012).

[16] D. W. Berry, M. Tsang, M. J. W. Hall, and H. M. Wiseman, Phys. Rev. X 5, 031018 (2015).

[17] Y.-R. Zhang and H. Fan, Phys. Rev. A 90, 043818 (2014).

[18] R. Nair, arXiv:1204.3761 [quant-th].

[19] V. Giovannetti, S. Lloyd, and L. Maccone, Phys. Rev. Lett. 108, 260405 (2012).
[20] V. Giovannetti and L. Maccone, Phys. Rev. Lett. 108, 210404 (2012).

[21] M. J. W. Hall, D. W. Berry, M. Zwierz, and H. M. Wiseman, Phys. Rev. A 85, 041802 (2012).

[22] M. J. W. Hall and H. M. Wiseman, Phys. Rev. X 2, 041006 (2012).

[23] M. J. W. Hall and H. M. Wiseman, New J. Phys. 14, 033040 (2012).

[24] H. L. Van Trees and K. L. Bell, eds., Bayesian Bounds for Parameter Estimation and Nonlinear Filtering/Tracking (Wiley-IEEE, Piscataway, New York, 2007).

[25] J. Ziv and M. Zakai, IEEE Trans. Inform. Theor. 15, 386 (1969).

[26] A. Weiss and E. Weinstein, IEEE Trans. Inform. Theory 31, 680 (1985).

[27] E. Weinstein and A. Weiss, IEEE Trans. Inform. Theory 34, 338 (1988).

[28] D. Nicholson and A. Vecchio, Phys. Rev. D 57, 4588 (1998).

[29] M. Tsang, H. M. Wiseman, and C. M. Caves, Phys. Rev. Lett. 106, 090401 (2011).

[30] Y. Tsuda and K. Matsumoto, J. Phys. A: Math. Gen. 38, 1593 (2005).

[31] S. L. Braunstein and C. M. Caves, Phys. Rev. Lett. 72, 3439 (1994).

[32] F. Hiai, M. Mosonyi, D. Petz, and C. Bény, Rev. Math. Phys. 23, 691 (2011).

[33] K. M. R. Audenaert, J. Calsamiglia, R. Muñoz Tapia, E. Bagan, L. Masanes, A. Acin, and F. Verstraete, Phys. Rev. Lett. 98, 160501 (2007).

[34] M. Nussbaum and A. Szkoa, Ann. Statist. 37, 1040 (2009).

[35] K. Audenaert, M. Nussbaum, A. Szkoa, and F. Verstraete, Commun. Math. Phys. 279, 251 (2008).

[36] K. Macieszczak, M. Fraas, and R. DemkowiczDobrzański, New J. Phys. 16, 113002 (2014).

[37] M. Jarzyna and R. Demkowicz-Dobrzanski, New J. Phys. 17, 013010 (2015).

[38] I. Bengtsson and K. Życzkowski, Geometry of Quantum States (Cambridge University Press, Cambridge, 2006). 